OPEN ACCESS

Edited by:

Imrich Barak

Slovak Academy of Sciences, Slovakia

Reviewed by:

Daniel Paredes-Sabja, Universidad Andres Bello, Chile

Christophe Nguyen-The,

Institut National de la Recherche

Agronomique, France

*Correspondence:

Oscar P. Kuipers

O.P.Kuipers@rug.n

${ }^{\dagger}$ Present Address:

Robyn T. Eijlander,

NIZO Food Research, Ede,

Netherlands

Specialty section:

This article was submitted to Microbial Physiology and Metabolism,

a section of the journal

Frontiers in Microbiology

Received: 12 August 2016 Accepted: 26 September 2016

Published: 13 October 2016

Citation:

Eillander RT, Holsappel S, de Jong A, Ghosh A, Christie G and Kuipers OP (2016) SpoVT: From Fine-Tuning Regulator in Bacillus subtilis to Essential Sporulation Protein in

Bacillus cereus.

Front. Microbiol. 7:1607. doi: 10.3389/fmicb.2016.01607

\section{SpoVT: From Fine-Tuning Regulator in Bacillus subtilis to Essential Sporulation Protein in Bacillus cereus}

\author{
Robyn T. Eijlander ${ }^{1,2+}$, Siger Holsappel ${ }^{2}$, Anne de Jong ${ }^{1,2}$, Abhinaba Ghosh $^{3}$, \\ Graham Christie ${ }^{3}$ and Oscar P. Kuipers ${ }^{1,2 *}$
}

${ }^{1}$ Top Institute Food and Nutrition, Wageningen, Netherlands, ${ }^{2}$ Department of Molecular Genetics, Groningen Biomolecular Sciences and Biotechnology Institute, University of Groningen, Groningen, Netherlands, ${ }^{3}$ Department of Chemical Engineering and Biotechnology, Institute of Biotechnology, University of Cambridge, Cambridge, UK

Sporulation is a highly sophisticated developmental process adopted by most Bacilli as a survival strategy to withstand extreme conditions that normally do not support microbial growth. A complicated regulatory cascade, divided into various stages and taking place in two different compartments of the cell, involves a number of primary and secondary regulator proteins that drive gene expression directed toward the formation and maturation of an endospore. Such regulator proteins are highly conserved among various spore formers. Despite this conservation, both regulatory and phenotypic differences are observed between different species of spore forming bacteria. In this study, we demonstrate that deletion of the regulatory sporulation protein SpoVT results in a severe sporulation defect in Bacillus cereus, whereas this is not observed in Bacillus subtilis. Although spores are initially formed, the process is stalled at a later stage in development, followed by lysis of the forespore and the mother cell. A transcriptomic investigation of $B$. cereus $\Delta$ spoVT shows upregulation of genes involved in germination, potentially leading to premature lysis of prespores formed. Additionally, extreme variation in the expression of species-specific genes of unknown function was observed. Introduction of the B. subtilis SpoVT protein could partly restore the sporulation defect in the $B$. cereus spoVT mutant strain. The difference in phenotype is thus more than likely explained by differences in promoter targets rather than differences in mode of action of the conserved SpoVT regulator protein. This study stresses that evolutionary variances in regulon members of sporulation regulators can have profound effects on the spore developmental process and that mere protein homology is not a foolproof predictor of similar phenotypes.

Keywords: sporulation, germination, gene regulation, Bacillus cereus, SpoVT

\section{INTRODUCTION}

Strains of Bacillus species are able to form endospores as a survival strategy in response to poor growth conditions. Their metabolic inactive state and sophisticated layered structures lead to strong resistance properties that enable the spore to survive conditions of increased heat, UV radiation, acid concentrations, pressure or low levels of water, or nutrients for extremely long periods of time (Setlow, 2006, 2007; Sella et al., 2014), while maintaining the ability to monitor their surroundings 
and respond to improvements through the process of germination and outgrowth (Setlow, 2003, 2013; Moir, 2006; Dworkin and Shah, 2010). Sporulation, spore resistance development, spore germination, and spore outgrowth are processes that are characterized by inter-strain and intra-strain heterogeneity and variety. This hampers the eradication of spores from food products or raw ingredients as it complicates predictability of spore properties and behavior (Cronin and Wilkinson, 2008; Augustin, 2011; Eijlander et al., 2011). Returning to a vegetative state, germinated spores are a major cause of food spoilage and of food poisoning (in the case of toxin production) (Brown, 2000; Abee et al., 2011; Logan, 2012).

Dormant spores are the final result of sporulation, which involves complex gene regulatory processes taking place in two different compartments of the cell (Eijlander et al., 2014). Sporulation-specific sigma factors govern the expression of dedicated gene sets in various stages, a process that is regulated in a sequential fashion (Hilbert and Piggot, 2004). The early regulator proteins SpoOA and $\sigma^{\mathrm{H}}$ are responsible for the consecutive expression and activation of $\sigma^{\mathrm{F}}$ in the forespore and $\sigma^{\mathrm{E}}$ in the mother cell (Yudkin and Clarkson, 2005). Expression and activation of late-stage sporulation sigma factors $\sigma^{\mathrm{G}}$ in the forespore and $\sigma^{\mathrm{K}}$ in the mother cell depend on completion of these earlier sporulation stages (Li and Piggot, 2001; Chary et al., 2005) (Figure 1). Binding of each sigma factor to RNA polymerase leads to interaction with dedicated DNA targets resulting in the spatial and temporal expression of specific sporulation gene sets required for the development and assembly of the forespore (Rudner and Losick, 2001; Hilbert and Piggot, 2004). Most proteins playing key roles in germination are produced during the later stages in sporulation.

The expression levels of sporulation and germination genes are fine-tuned by secondary regulator proteins that are under the control of sigma factors and enable feed forward loops in the regulatory system. One of these proteins is SpoVT (Figure 1). Expressed in the forespore compartment during latestage sporulation under the control of $\sigma^{\mathrm{G}}$, SpoVT enhances the expression of some $\sigma^{\mathrm{G}}$-dependent genes and represses others (Bagyan et al., 1996). For DNA binding it forms a tetramer and possibly binds a yet unidentified substrate through means of the GAF domain in the C-terminal part of the protein (Dong et al., 2004; Asen et al., 2009). Deletion of spoVT from B. subtilis cells results in the formation of spores that have a defective spore coat, a faster nutrient-induced germination response, an increased sensitivity to UV radiation and limited ability for spore outgrowth (Bagyan et al., 1996; Ramirez-Peralta et al., 2012). A recent study in B. subtilis has shown that levels of active SpoVT play a crucial role in determining the numbers of important germination proteins, such as nutrient germination receptors (GRs) and small acid-soluble proteins (SASPs), which affects the germination, resistance, and outgrowth properties of the spore (Ramirez-Peralta et al., 2012).

The SpoVT protein is highly conserved amongst sporeforming bacteria, especially Bacilli (Asen et al., 2009; RamirezPeralta et al., 2012). The extreme sequence similarity ( $95 \%)$ of the N-terminal domain of SpoVT homologs suggests a similar mode of action concerning DNA binding activity. It must be
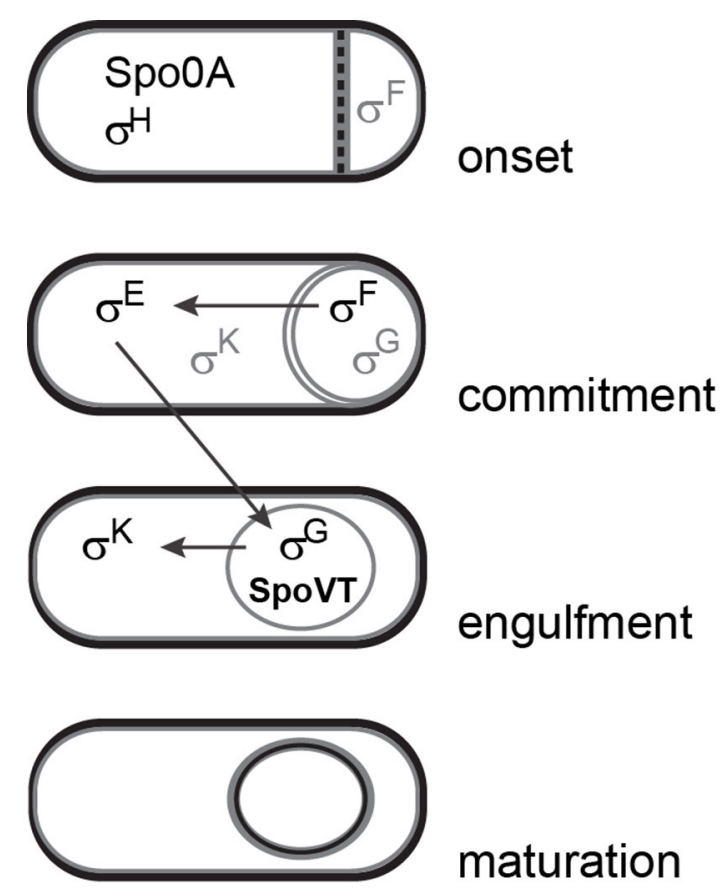

FIGURE 1 | Simplified schematic overview of different stages in sporulation and sequential activation of sporulation-specific sigma factors. Gray lettering indicates inactive forms of sigma factors. This figure was adapted from Piggot and Hilbert (2004).

noted, however, that so far no consensus binding site for SpoVT has been identified (Dong et al., 2004). The C-terminal domain is less conserved (sequence identity of 20-30\%) and shows features of a GAF domain that resembles the one of CodY (Asen et al., 2009). Such domains harbor binding pockets for the binding of substrates that are required for structure, sensing, and/or activity. If SpoVT requires substrate-binding for activity, it is possible that this substrate differs amongst various SpoVT homologs (Asen et al., 2009). However, several attempts to identify such a substrate have so far failed.

The SpoVT protein of Bacillus cereus (from here on referred to as $\left.\mathrm{SpoVT}_{\mathrm{BCE}}\right)$ has been less well studied than its $B$. subtilis counterpart and its precise role in gene expression during sporulation is so far unknown. $B$. cereus is notorious for compromising food quality and safety due to its high diversity, the production of highly heat-resistant spores and cytotoxinproducing properties (Stenfors Arnesen et al., 2008; Lücking et al., 2013). It is part of a wider class of related species that also includes Bacillus anthracis, the causer of anthrax, and Bacillus thuringiensis, an insect pathogen. The main characteristics of these closely related species are determined by large plasmids that encode specific toxin or other virulence factors (Økstad and and Kolstø, 2011). The non-pathogenic B. subtilis lacks such plasmids and is part of a different group of Bacilli that also includes Bacillus licheniformis and Bacillus amyloliquefaciens. Due to a wealth of available experimental data, B. subtilis and $B$. cereus are commonly used as model organisms for studies concerning these two groups of species, with in particular $B$. 
subtilis 168 and B cereus ATCC 14579. Despite a common ability to form endospores and a conservation of important sporulation and germination genes (de Vries et al., 2004; de Hoon et al., 2010; Galperin et al., 2012), interesting evolutionary diversity can be observed between these two species. This is reflected by differences in gene regulation during sporulation (Pflughoeft et al., 2011) or in various spore properties, such as the spore coat (Wang et al., 2007; Qin and Driks, 2013), spore size (Carrera et al., 2007), germinant receptor protein types (Hornstra et al., 2005; van der Voort et al., 2010), and spore resistance properties (Wang et al., 2003; Black et al., 2008).

In this study, we investigated the role or $\mathrm{SpOVT}_{\mathrm{BCE}}$ in (heterogeneous) gene expression during sporulation of B. cereus. We show that, in contrast to what was previously reported for B. subtilis, deletion of spoVT $T_{\mathrm{BCE}}$ results in a complete sporulation defect. Transcriptomic investigation during sporulation shows upregulation of genes involved in germination, which indicates premature lysis of prespores. In addition, the data shows differential expression of $B$. cereus-specific genes of unknown function, which potentially play an important role in the extreme phenotype. This is furthermore supported by successful complementation studies using both $\mathrm{SpoVT}_{\mathrm{BCE}}$ and $\mathrm{SpoVT}_{\mathrm{BSU}}$. Through this study we show that despite strong sequence conservation of SpoVT among Bacilli, significant differences exist in the role of this regulator during developmental processes, which are likely due to the specific genes under its control.

\section{MATERIALS AND METHODS}

\section{Strains and Plasmids}

All strains and plasmids used in this study are listed in Table $\mathbf{1 .}$ Primers used for the amplification of DNA fragments are listed in Table S1. As reference strains, B. subtilis 168 and B. cereus ATCC 14579 were used. Foreign DNA was introduced into $B$. cereus via electroporation (Masson et al., 1989), either by using the multicopy plasmid pNW33n or by single crossover DNA integration via non-replicative pMAD and PSG1151 derivatives.

For the construction of the pNWVT vector, the spoVT gene (BC0059) including its own promoter was amplified from $B$. cereus ATCC 14579 chromosomal DNA using primers AKupVT-F and TIFN16. The resulting product was cut with EcoRI and KpnI (Fermentas, FastDigest) and ligated into the corresponding sites of pNW33n (Genbank Accession number, AY237122), which resulted in pNWVT. The pVTBsu2 vector was created by replacing $s p o V T_{\mathrm{BCE}}$ with $s p o V T_{\mathrm{BSU}}$ in pNWVT, which was amplified using primers BsuVTF2 and BsuVTR. The primers were specifically designed to ensure that spoVT $T_{\mathrm{BSU}}$ expression would be driven by the $B$. cereus spoVT promoter already present in pNWVT. The spoVT $\mathrm{BCE}$ gene was cut out of pNWVT using BclI and HindIII and replaced with spoVT $T_{\mathrm{BSU}}$ containing compatible sticky ends. Correct construction was verified using restriction analysis and sequencing.

For the construction of the B. cereus spoVT disruption mutant, we constructed the pDCVT vector by amplifying an upstream spoVT flanking region from B. cereus ATCC $14579 \mathrm{gDNA}$ using primers dCVT-F1 and dCVT-R1. A downstream flanking region was amplified using primers dCVT-F2 and dCVT-R2. Both flanking regions were fused to HindIII compatible ends of a spectinomycin resistance cassette originating from pDG1726. The fused fragments were ligated into the NcoI and EcoRI sites of pMAD to create pDCVT. The pDCVT was introduced into B. cereus ATCC 14579 to disrupt the spoVT gene according to the method described by Arnaud et al. (2004). Disruption of spoVT

TABLE 1 | Strains and plasmids used in this study.

\begin{tabular}{|c|c|c|}
\hline Strain & Properties & References \\
\hline B. subtilis 168 & $\operatorname{trpC2}$ & Kunst et al., 1997 \\
\hline B. subtilis $168 \Delta$ spovT & spoVt::spec ${ }^{r}$ & $\begin{array}{l}\text { Bagyan et al., 1996, kindly provided by Prof. S. } \\
\text { Cutting }\end{array}$ \\
\hline B. cereus ATCC 14579 & Enterotoxic strain of $B$. cereus wild type isolate & $\begin{array}{l}\text { Bacillus Genetic Stock Center, ATCC, BGSC } \\
\text { ID6A5 }\end{array}$ \\
\hline B. cereus ATCC $14579 \mathrm{P}_{\text {spova-gfp }}$ & 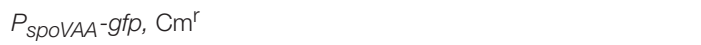 & This study \\
\hline B. cereus ATCC $14579 \Delta$ spoVT & spoVT::spec ${ }^{r}$ & This study \\
\hline B. cereus ATCC $14579 \Delta$ spoVT $P_{\text {spova-gfp }}$ & spoVT::spec ${ }^{r}, P_{\text {spoVAA-gfp }} \mathrm{Cm}^{r}$ & This study \\
\hline B. cereus ATCC $14579 \Delta \mathrm{BC} 1117$ & marker-less deletion of the $\mathrm{BC} 1117 \mathrm{ORF}$ & This study \\
\hline Plasmid & Properties & References \\
\hline pNW33n & E. coli-Gram + shuttle vector & Bacillus Genetic Stock Center \\
\hline pNWVT & $\mathrm{Cm}^{r}$, spoVt $\mathrm{BCE}$ & This study \\
\hline pVTBsu2 & $\mathrm{Cm}^{r}$, spoVt $T_{\mathrm{BSU}}$ & This study \\
\hline pSG1151 & Vector for integrative P-gfpmut1 fusions in B. subtilis, $\mathrm{Ap}^{r}, \mathrm{Cm}^{r}$ & Lewis and Marston, 1999 \\
\hline pSGCVA & pSG1151 with gfp driven by the Bce spoVAA promoter, $\mathrm{Ap}^{r}, \mathrm{Cm}^{r}$ & This study \\
\hline PMAD & $\begin{array}{l}\text { Vector for efficient gene replacement in non-naturally } \\
\text { transformable gram-positive bacteria, } \mathrm{Ap}^{r}, \mathrm{Em}^{r}\end{array}$ & Arnaud et al., 2004 \\
\hline pDCVT & pMAD-derivative used for spoVT gene disruption, $A p^{r}, E m^{r}$ & This study \\
\hline pDG1726 & E. coli plasmid bearing a spectinomycin resistance cassette & Guérout-Fleury et al., 1995 \\
\hline
\end{tabular}


was verified using PCR analysis and sequencing of the integration site to ensure there were no second-site mutations.

For the construction of a chromosomally integrated $\mathrm{P}_{\text {spoVA }}{ }^{-}$ $g f p$ fusion in B. cereus, a $1.5 \mathrm{~kb}$ fragment of the upstream region of the $B$. cereus ATCC 14579 spoVAA gene (BC4070) was amplified using primers TIFN41 and TIFN42A. The TIFN42A primer was designed as such that the original RBS plus the first two codons of the spoVAA gene were included in the amplified fragment. This was then cleaved with EcoRI and KpnI and introduced into the corresponding sites of pSG1151. The resulting pSGCVA vector was introduced into B. cereus ATCC 14579 and B. cereus ATCC $14579 \triangle$ spoVT via electroporation and checked for single crossover integration on the original locus using PCR analysis and sequencing.

A strain bearing an in-frame deletion of the ORF encoded at locus $\mathrm{BC} 1117$ was created using a marker-less gene replacement method (Janes and Stibitz, 2006; Lindbäck et al., 2012). Essentially, a modified pMAD-derived vector containing $\sim 500$ bp of the respective up- and downstream regions of DNA flanking the $\mathrm{BC} 1117 \mathrm{ORF}$ was introduced by electroporation to B. cereus ATCC 14579 cells. Plasmid pBKJ233, which encodes I-SceI enzyme, was introduced subsequently to cells identified by blue-white (X-gal) screening and PCR as having inserted the pMAD-derived plasmid at locus BC1117. Clones that had undergone a second recombination event, deleting the $\mathrm{BC} 1117$ ORF and leaving behind only the start and stop codons, were identified by screening for erythromycin sensitivity. Finally, the $\triangle \mathrm{BC} 1117$ strain was validated for the correct construction by PCR.

\section{Culture Preparation and Media}

All B. cereus strains were cultured at $30^{\circ} \mathrm{C}$ with aeration at $220 \mathrm{rpm}$. Media was supplemented with chloramphenicol (4 $\mu \mathrm{g} / \mathrm{ml})$, erythromycin $(2 \mu \mathrm{g} / \mathrm{ml})$, or spectinomycin $(300$ $\mu \mathrm{g} / \mathrm{ml}$ ) when appropriate. For the preparation of cells for timelapse microscopy, cells were prepared as previously described (Eijlander and Kuipers, 2013). For all other sporulation experiments and for the preparation of sporulating cells for RNA isolation, cells were cultured in maltose sporulation medium (MSM) (van der Voort et al., 2010). Spore crops were prepared on MSM agar plates for 7 days at $30^{\circ} \mathrm{C}$. Spores were collected from the plates and washed $\left(6000 \mathrm{rpm}, 15 \mathrm{~min}\right.$ at $\left.4^{\circ} \mathrm{C}\right)$ twice a day for 14 days in $10 \mathrm{mM}$ phosphate buffer ( $\mathrm{pH}$ 6.2) with decreasing concentrations of Tween 20 to prevent clumping of the spores $(0.1,0.075,0.05,0.04,0.03,0.02$, and $0.01 \%)$. Phase contrast microscopy analysis of the spore crops showed $>98 \%$ free-lying spores. Spores were stored at $4^{\circ} \mathrm{C}$ in $10 \mathrm{mM}$ phosphate buffer with $0.01 \%$ added Tween 20 and were washed twice a week to prevent spontaneous germination.

\section{Complementation of $\Delta$ spoVT with pNWVT (spoVt $T_{\mathrm{BCE}}$ ) and pVTBsu2 (spoVt $\left.T_{\mathrm{BSU}}\right)$}

The vectors pNWVT (containing spoVT $T_{\mathrm{BCE}}$ expressed by its own promoter) and $\mathrm{pVTBsu} 2$ (containing spoVT $T_{\mathrm{BSU}}$ expressed by the $\mathrm{P}_{\text {spoVTBCE }}$ promoter) were introduced in the B. cereus ATCC $14579 \Delta$ spoVT strain by electroporation as described above. Overnight cultures were diluted 1:100 in MSM medium and incubated at $30^{\circ} \mathrm{C}$ while shaking for $6 \mathrm{~h}$. Exponentially growing cells were again diluted 1:100 in fresh MSM medium and allowed to grow in the same conditions for $65 \mathrm{~h}$. Samples for phase contrast microscopy analysis were taken after 17, 22, 44, and $65 \mathrm{~h}$. Efficiency in complementation of sporulation was determined using the Cell Counter plugin in Image $)^{1}$.

\section{Germination Assays}

$B$. cereus spores were washed twice before the experiment and resuspended in ice-cold sterile demineralized water. Spores were heat-activated at $70^{\circ} \mathrm{C}$ for $15 \mathrm{~min}$ and immediately placed on ice for $2 \mathrm{~min}$ to cool down. The washing step was repeated after which spores were resuspended in ice-cold sterile germination buffer $(10 \mathrm{mM}$ Tris- $\mathrm{HCl} 7.4+10 \mathrm{mM} \mathrm{NaCl})$ to a final $\mathrm{OD}_{600}$ of 10. Spores were diluted 10 times in germination buffer containing nutrients $(10 \mathrm{mM}$ alanine or $1 \mathrm{mM}$ inosine) or BHI medium supplemented with chloramphenicol $(5 \mu \mathrm{g} / \mathrm{ml})$ to prevent outgrowth. Changes in optical density were monitored every $2 \mathrm{~min}$ for $4 \mathrm{~h}$ at $30^{\circ} \mathrm{C}$ in a TECAN plate reader and the obtained data plotted in Excel.

\section{Imaging, Time-Lapse Microscopy, and Image Analysis}

All microscopy imaging was performed using the IX71 Microscope (Olympus) with CoolSNAP HQ2 camera (Princeton Instruments) and DeltaVision softWoRx 3.6.0 (Applied Precision) software. For B. subtilis the $100 \mathrm{x}$ phase contrast objective was used, for B. cereus the $60 \mathrm{x}$ phase contrast objective. For the visualization of green fluorescence from GFP or FM46 dye the GFP filterset was used (Chroma, excitation at $470 / 40 \mathrm{~nm}$, emission at 525/50 nm). Images were taken using 32\% APLLC White LED light and $0.05 \mathrm{~s}$ exposure for bright field pictures and $10 \%$ Xenon light with $0.5 \mathrm{~s}$ exposure for fluorescence detection. Pictures were analyzed using ImageJ software.

To monitor gene expression in single cells during sporulation, the time-lapse microscopy technique was applied for the promoter- $g f p$ fusion strains of $B$. cereus as previously described (Eijlander and Kuipers, 2013). For quantification of the GFP fluorescence signal distribution, the fluorescence intensity was measured for every cell in the microcolony for every time frame using the ImageJ ROI tool. Fluorescence intensities per cell per frame were exported to Excel and normalized by subtracting background fluorescence levels of the microscopy slide (agarose) and autofluorescence of the cells or spores (highest fluorescent value measured for wt strains). Mean fluorescence values were calculated from ten frames per cell and binned according to set fluorescence value categories. This data was represented in fluorescence distribution plots in Excel.

\section{Total RNA Extraction from Sporulating B. cereus Cells}

Cells were cultured in sporulation media to induce the sporulation event. Individual stages in sporulation were determined via fluorescence microscopy on agarose patches with

\footnotetext{
${ }^{1}$ http://imagej.nih.gov/ij/.
} 
added $\mathrm{FM}^{\circledR}$ 1-43 membrane stain (Invitrogen, Ex 479 nm, Em $598 \mathrm{~nm})$ at an end concentration of $1 \mu \mathrm{g} / \mu \mathrm{l}$.

Cell samples $(5 \mathrm{ml}$, including biological and technical replicates) were taken every hour for $6 \mathrm{~h}$ from transition point onwards (T0, reached after $7 \mathrm{~h}$ of growth in MSM). Cells were collected by centrifugation in a pre-cooled centrifuge $\left(4^{\circ} \mathrm{C}, 4000\right.$ $\mathrm{rpm}, 3 \mathrm{~min}$ ) and immediately frozen in liquid nitrogen. For RNA extraction, cells were thawed on ice in $500 \mu \mathrm{l}$ TRI-reagent (Life Technologies, Carlsbad, CA USA) and glass beads $(<100 \mu \mathrm{m}$, Sigma-Aldrich), resuspended and immediately disrupted by 4 rounds of bead beating [ $45 \mathrm{~s}$ at maximal settings $(3450 \mathrm{rpm})$ ] in a Mini-Beadbeater-16 (BioSpec products, Bartlesville, OK USA). Direct-zol RNA MiniPrep (Zymo Research, Irvine, CA USA) was used according to manufacturer's instruction for on column RNA purification. Residual chromosomal DNA was removed using the Ambion DNA-free ${ }^{\mathrm{TM}}$ kit (Life Technologies ${ }^{\circledR}$, Thermo Fisher Scientific, USA). The RNA concentration was measured on a NanoDrop ND-1000 spectrophotometer. RNA quality was determined using an Agilent BioAnalyzer RNA 6000 nanokit. The concentration of RNA isolated from wt sporulation cells was a little lower than for $\Delta s p o V T$ sporulating cells $(3000 \mathrm{ng} / \mu \mathrm{l}$ compared to $3800 \mathrm{ng} / \mu \mathrm{l}$ ) but still comparable. Quality of all isolations was within the specified levels for all samples (an $\mathrm{OD}_{260 / 280}$ ratio between 1.8 and 2.0 and an $\mathrm{OD}_{260 / 230}$ ratio of $>1.7)$.

\section{RNA Sequencing}

RNA samples $(>1 \mu \mathrm{g})$ were sent to PrimBio Research Institute (Exton, PA, USA) where rRNA depletion (using the Ambion MICROBExpress ${ }^{\mathrm{TM}} \mathrm{Kit}$ ) and a library prep (using the Ion Total RNA-Seq Kit v2) were performed. Samples were multiplexed in sets of nine and loaded on an Ion Proton ${ }^{\mathrm{TM}}$ chip. In total $80 \mathrm{M}$ reads with an average length of 120 bases were derived per chip. The reads were mapped against the reference genomes for B. cereus ATCC 14579 using Bowtie 2 (Langmead and Salzberg, 2012) with optimized parameters setting for Ion Proton data: “-D 20 -R 3 -N 1 -L 20 -i S, 1, 0.50 -local”. Gene expression profiling was done using SAMtools (Li et al., 2009) and Cufflinks (Trapnell et al., 2012). Subsequent statistical analysis was performed using a modified EdgeR routine on the MolGen webserver ${ }^{2}$.

Spearman Rank correlation analysis was performed to show clustering of biological replicates within one strain and between strains. Correctly clustered data sets were further filtered based on intra-variation < inter-variation. Excel conditional formatting was applied to visualize the degree of intra- and inter-variation for each differentially expressed gene. The biological ratio in gene expression was calculated using log-transformed data in the following formula:

$$
\text { Biological ratio }=\log 2(w t 1+w t 2)-\log 2(d V T 1+d V T 2)
$$

where $w t 1$ and $w t 2$ are replicate rpkm values for the $w t$ strain and $d V T 1$ and $d V T 2$ are replicate rpkm values for the $\Delta s p o V T$ strain. The fold change in gene expression between the two strains was furthermore determined by raising the number 2 to the power of the absolute value for the ratio $\left(2^{\mathrm{ABS}(\text { ratio) }}\right)$.

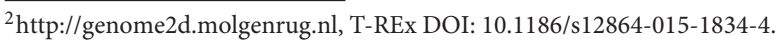

Orthologs of B. subtilis sporulation genes (SporeWeb $^{3}$, Eijlander et al., 2014) in B. cereus ATCC 14579 were determined using OrthoMCL (Chen et al., 2006) and (predicted) gene function using the KEGG database ${ }^{4}$ or NCBI Blast ${ }^{5}$.

\section{RESULTS}

\section{spoVT Is Essential for Completion of Sporulation in Bacillus cereus}

All present knowledge on the SpoVT regulator protein so far originates from studies in B. subtilis. Cells of B. subtilis that lack SpoVT are still able to produce spores, albeit with a defective spore coat structure, an increased initial germination rate in response to nutrients and a severe defect in further germination and outgrowth (Bagyan et al., 1996; RamirezPeralta et al., 2012). Despite a strong conservation of SpoVT in endospore-forming bacteria, the effect of removing SpoVT from B. cereus cells (SpoVT $\mathrm{BCE}$ ) proves more severe. In this organism, a similar spoVT disruption mutant is unable to complete the sporulation process and produces very few, if any, free-lying spores with a severely defective morphology (Figure $2 \Delta s p o V T$ ). Re-introduction of spoVT $T_{\mathrm{BCE}}$ only partly restored this defect (14\% of the total number of cells restored sporulation, Figure 2 $\Delta s p o V T+)$. The partial complementation result could be due to differences in plasmid copy number between individual spores. Interestingly, similar complementation experiments with the spoVT wt allele of B. subtilis (spoVT $T_{\mathrm{BSU}}$ driven by the PspoVT $T_{\mathrm{BCE}}$ promoter) produced comparable results (complementation restored sporulation in $12 \%$ of the total number of cells, Figure 2, $\Delta s p o V T+\mathrm{Bsu})$. This indicates that the working mechanism of SpoVT $\mathrm{BSU}$ and $\mathrm{SpoVT}_{\mathrm{BCE}}$ is comparable, which is expected given the high sequence conservation between both proteins. The observed difference in phenotypic effect after removal of SpoVT in both organisms must thus have a basis in the promoters of these regulatory proteins interact with to modulate gene expression.

\section{Sporulation of a spoVT Deletion Strain of $B$. cereus Is Stalled at a Later Stage Leading to Premature Lysis}

Time-lapse fluorescence microscopy is a powerful technique to visualize dynamics in gene expression in time in individual cells and was recently optimized for application with $B$. cereus cells (Eijlander and Kuipers, 2013). In this study, we applied timelapse fluorescence microscopy to follow the events of foresporespecific gene expression in time in a spoVT deletion background of $B$. cereus. Unfortunately, multiple attempts to construct a variety of sporulation-specific promoter- $g f p$ fusions (e.g., $\mathrm{P}_{\text {gerI }}$, $\mathrm{P}_{c w l}, \mathrm{P}_{\text {gerR }}, \mathrm{P}_{\text {cotD }}, \mathrm{P}_{\text {sspA }}$ ) stably integrated into the $B$. cereus genome failed, except for one (i.e., $\mathrm{P}_{s p o V A}-g f p$ ). This limited our ability to determine true effects of SpoVT removal on the expression levels of late sporulation genes, but did enable us to

\footnotetext{
${ }^{3}$ http://sporeweb.molgenrug.nl.

${ }^{4}$ www.genome.jp/kegg/.

${ }^{5}$ http://blast.ncbi.nlm.nih.gov/.
} 

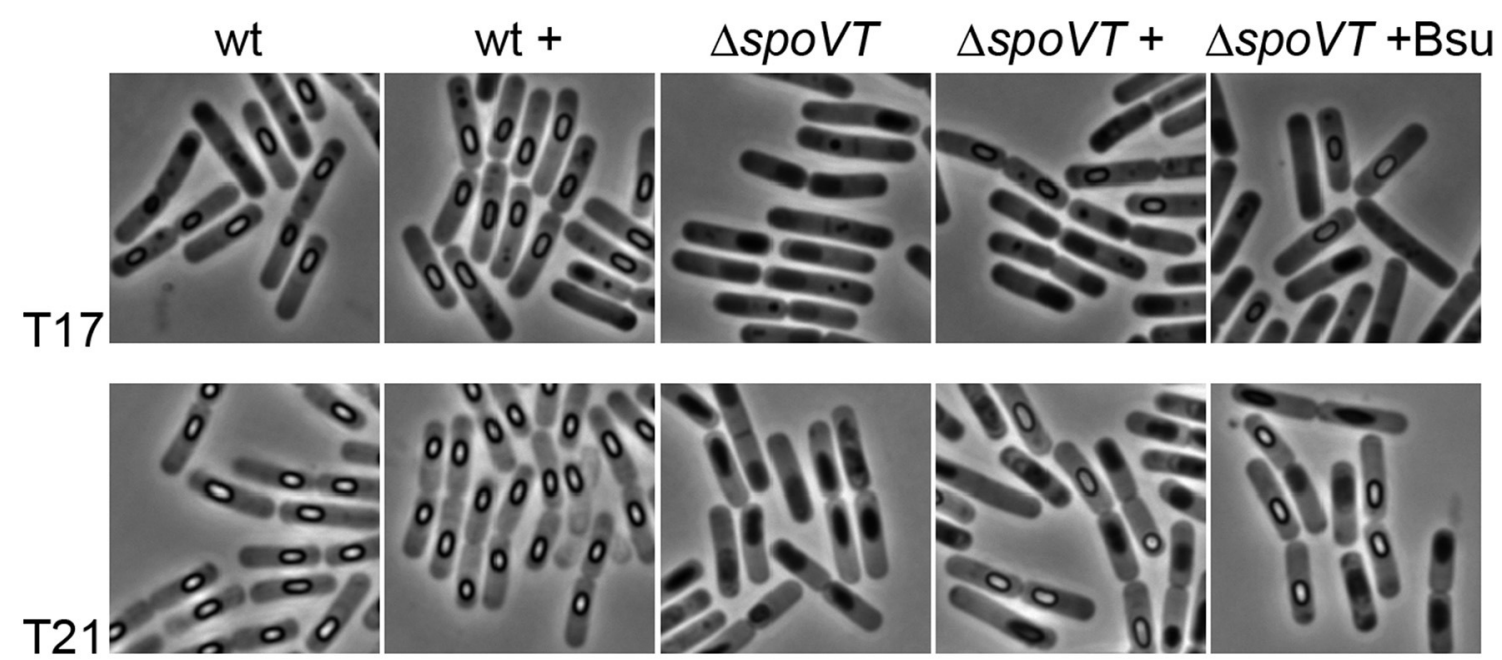

T44
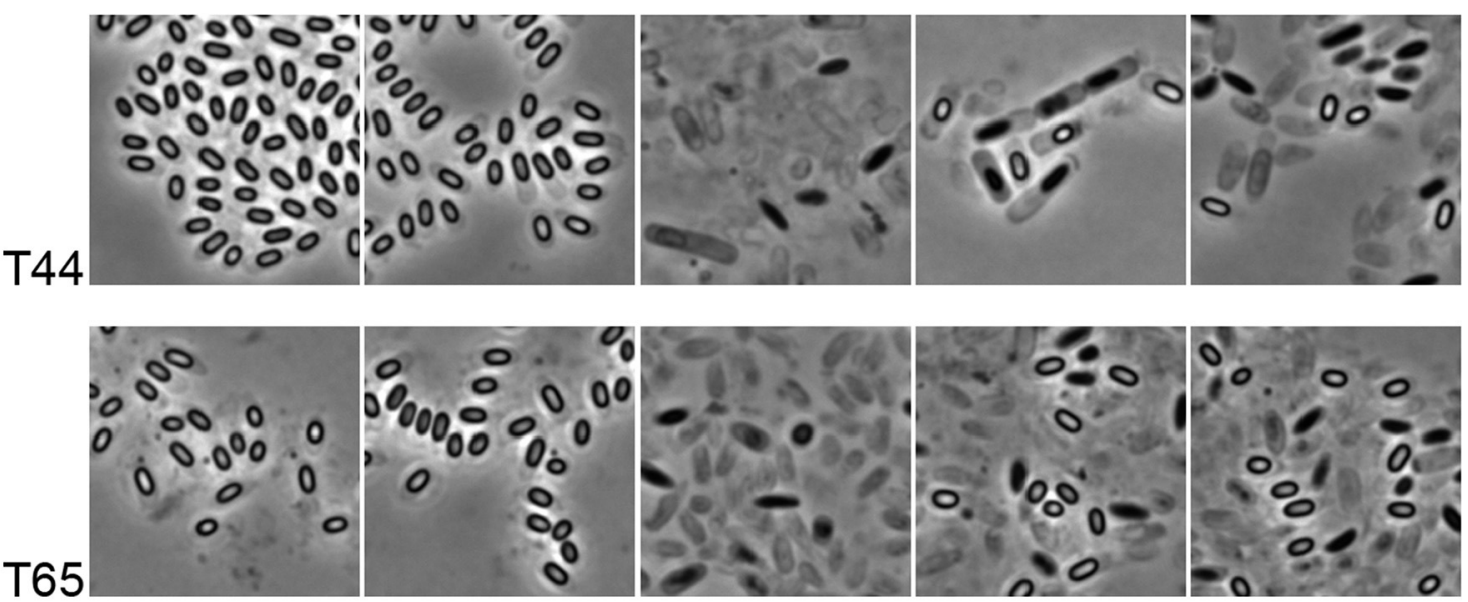

FIGURE 2 | Sporulation of B. cereus in the presence and absence of SpoVT. Sporulation was induced by growth in MSM medium and the progress of sporulation was monitored using phase-contrast microscopy for the B. cereus ATCC 14579 wild type strain (wt) and spoVT deletion strain ( $\Delta$ spoVT) with plasmid-borne spoVt $T_{\mathrm{BCE}}(+)$ or spoVt $T_{\mathrm{BSU}}(+\mathrm{Bsu})$ (originating from the pNWVT or pVTBsu2 vector, respectively). The B. cereus wt strain with extra plasmid-borne spoVt $\mathrm{BCE}(\mathrm{Wt}+)$ was added as a control to ensure no unexpected sporulation defects were occurring due to pNWVT-derived spoVT expression during sporulation. Samples were analyzed after $17,21,44$, and $65 \mathrm{~h}$ of growth after initial dilution in fresh medium.

provide insights in the sporulation line of events in the absence of SpoVT.

Time-lapse image analysis of strain B. cereus $\Delta s p o V T$

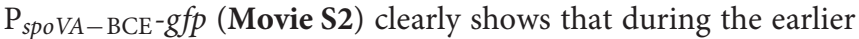
stages of sporulation prespores are formed like in the wild type background (Movie S1), but that progression of sporulation is stalled at a later stage. First, the formed pre-spores lyse, thereby spreading the produced GFP throughout the mother cell (Figure 3B and Movie S2). Finally, the mother cell also lyses (Figure 3A).

A quantitative analysis of the obtained images was performed to substantiate the difference in spoVA gene expression levels in a spoVT background. Before pre-spore lysis, the intensity of fluorescence originating from the $\mathrm{P}_{s p o V A} g f p$ fusion was significantly higher in the absence of SpoVT $\mathrm{BCE}_{\mathrm{B}}$ than in wild type cells (Figure 3C and Movies S1, S2). In addition, the distribution of the signal strength was more wide-spread amongst individual cells. It must be noted, however, that it is possible that this observed effect is not the result of increased (heterogeneity in) promoter activity in the absence of SpoVT, but rather an artifact in the detection of the GFP signal-the spoVT spores are severely weakened shortly after the completion of engulfment and, furthermore, it is known that SpoVT enhances rather than represses spoVA expression in B. subtilis (Wang et al., 2006; Ramirez-Peralta et al., 2012).

\section{Transcriptomic Analysis of B. cereus $\Delta$ spoVT during Sporulation}

To further investigate the role of SpoVT in gene expression during sporulation of $B$. cereus, we isolated RNA from sporulating cells at three sequential time points $(3,4$, and $5 \mathrm{~h}$ after the transition point to stationary growth was reached; Figure S1). Differentially expressed genes in the spoVT deletion strain were compared to the wild type situation using a 
A B. cereus ATCC14579 PspovA-gfp

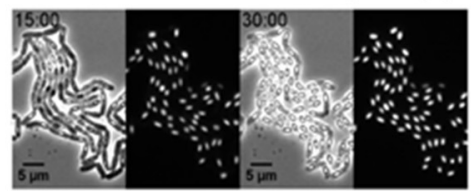

B. cereus ATCC14579 $\Delta$ spoVT PspoVA-gfp

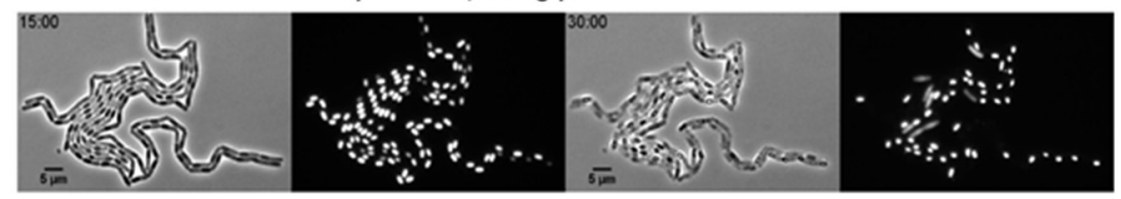

B

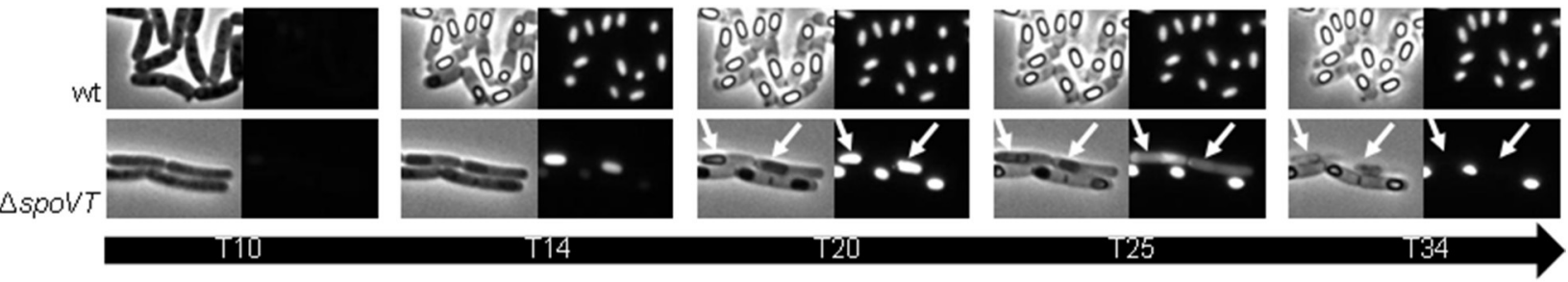

C

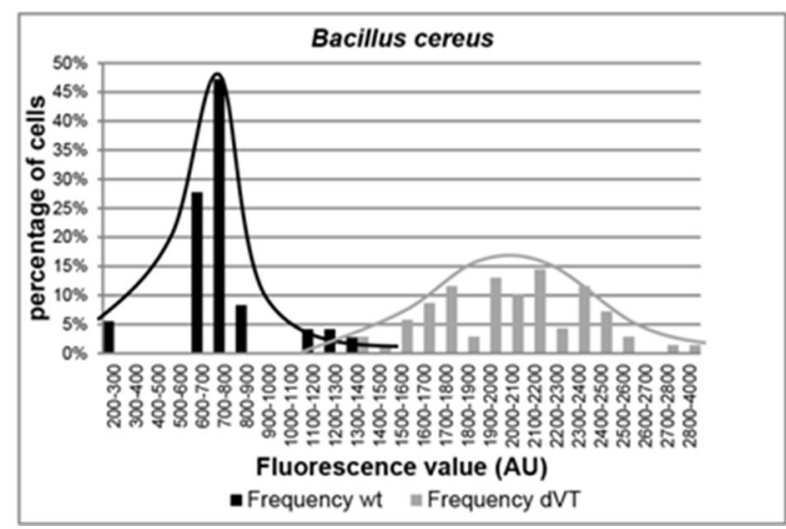

FIGURE $3 \mid \mathbf{P}_{\text {spoVA }}$ promoter activity and signal distribution in a wt and spoVT deletion strain of $\boldsymbol{B}$. cereus. $\mathrm{P}_{\text {spoVA }}$ promoter activity was measured in the wild type strain (B. cereus ATCC14579) and a $\Delta$ spoVT background using time-lapse microscopy. (A) Two time frames from movies S1 (wt) and S2 ( $\Delta$ spoVT) show

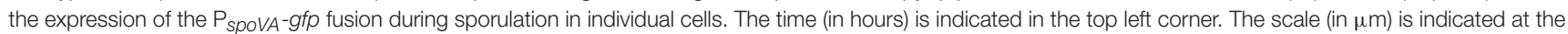
bottom left corner. (B) Cut-outs of individual cells during five different time points (in hours) from Movies S1, S2 are shown to visualize the line of events during sporulation in a wt and a $\Delta s p o V T$ background. Lysing prespores and mothercells in $\Delta s p o V T$ are indicated by white arrows. (C) The average fluorescence value distribution in arbitrary units in B. cereus wt (black bars) and spoVT cells (gray bars) calculated from Movies S1, S2.

transcriptomics approach. Details on RNA isolation, preparation, sequencing, downstream normalization, and statistical analysis of the resulting data are described in the Materials and Methods Section. Resulting normalized expression values [reads per kilobase per million $(\mathrm{rpkm})$ ] are provided in Table S2.

Sporulation is a very heterogeneous process, which complicates the synchronization of gene expression in individual cells. This generates a large variety in the absolute gene expression values generated by RNA sequencing. Spearman Rank correlation of the available data showed that two biological replicates of the same strain (wt or $\Delta s p o V T$ ) clustered together only for the samples taken at T5. Therefore, further data analysis was performed for that time point only.

The log2-transformed data was used to calculate the fold change in gene expression in the spoVT mutant strain compared to the wt strain. A total list of significantly up- and downregulated genes is provided in Table S3.
Of the differentially expressed genes (with a cut-off of $\geq 2$-fold difference), a total number of 52 genes were down-regulated and 87 genes were up-regulated (Table 2). Both groups contain genes involved in sporulation, germination, regulation, metabolism, and transport and both groups contain a large number of genes encoding hypothetical or unknown proteins. What stands out, is that genes involved in protein synthesis (ribosomal protein subunits) were specifically upregulated in a spoVT mutant. In addition, a large number of genes involved in metabolism was also upregulated.

Concerning sporulation and/or germination-specific genes, almost no $B$. cereus orthologs of known SpoVT-regulated genes (as determined by studies in B. subtilis; Bagyan et al., 1996; Wang et al., 2006) are present in this list. Only spoIIIG (BC3903) and tepA (BC3795) were, similarly to their counterparts in B. subtilis, repressed by SpoVT in B. cereus (Table S3). Amongst significantly down-regulated sporulation genes ( $>2$-fold difference in gene expression) were some genes 
TABLE 2 | Number of genes per functional category up-or down-regulated in a $B$. cereus spoVT deletion background.

\begin{tabular}{lcc}
\hline Functional category & $\begin{array}{c}\text { Number of genes } \\
\text { upregulated }\end{array}$ & $\begin{array}{c}\text { Number of genes } \\
\text { downregulated }\end{array}$ \\
\hline Sporulation & 15 & 9 \\
Germination & 4 & 1 \\
Metabolism and transport & 25 & 10 \\
Protein synthesis & 13 & 0 \\
Regulation & 6 & 4 \\
Hypothetical and unknown & 16 & 20 \\
Other & 8 & 8 \\
\hline
\end{tabular}

encoding spore coat proteins, one germination receptor subunit (GerIA) and a few sporulation proteins of unknown function. Upregulated sporulation genes, on the other hand, were those encoding CwlJ1, SpoIIQ, SpmA, CotD, YaaH, SpoIVA, SpoIIIAH, CdaS, SpoIIIAC, genes encoding sporulation-specific regulators RsfA, SigK, GerE, SigG, and AbrB and a few sporulation proteins of unknown function.

Interestingly, quite a few hypothetical or genes of unknown function were extremely, or at least considerably, downregulated in the spoVT deletion strain (Table 3). Although no putative function could be deducted from homology searches using their protein sequences, most of them seem to be specific for $B$. cereus group organisms. One such example is the gene encoded at locus $\mathrm{BC} 1117$, expression of which was down-regulated approximately 3000 -fold in the spoVT mutant strain. BC1117 is predicted to encode a small (50 residue, $\sim 6 \mathrm{KDa}$ ) lysine-rich polypeptide. A preliminary examination of the role of BC1117 in B. cereus ATCC 14579 was conducted by constructing a strain with a markerless deletion in this locus. However, in contrast to the $\Delta s p o V T$ strain, the $\triangle \mathrm{BC} 1117$ strain was observed to grow and sporulate normally, releasing mature spores after approximately $24 \mathrm{~h}$ of culture (Figure S2).

\section{DISCUSSION}

SpoVT is a strongly conserved regulatory protein specifically active during the later sporulation stages in the forespore compartment of cells and is known to play an important role in fine-tuning the regulation of sporulation-specific gene expression (Ramirez-Peralta et al., 2012). The lack of a consensus SpoVT binding sequence complicates the prediction of alternating SpoVT level effects on spore properties and spore germination efficiency. The strongly conserved N-terminal DNA-binding domain of SpoVT suggests a similar conservation in DNA targets for this regulatory protein. However, in this work we show that removing SpoVT from sporulating cells has a dramatic effect on completion of sporulation in B. cereus-an effect that is not observed in B. subtilis. Unfortunately, we were unable to study single copy integrated SpoVT complementation due to the low efficiency of DNA recombination in B. cereus (Arnaud et al., 2004). By making use of a vector-based complementation, restoration of the sporulation phenotype was only partly accomplished. This could be due to differences in plasmid copy number between individual spores (Turgeon et al., 2008), which would suggest that the tolerated levels of SpoVT $\mathrm{BCE}$ protein in sporulating cells fit inside a tight window.

Furthermore, similar complementation results with heterologous SpoVT $\mathrm{BSU}_{\mathrm{BS}}$ indicate that the observed difference in the impact of SpoVT removal from sporulating cells of the two species is not due to a difference in the working mechanism, but rather to a difference in regulated target genes. This is supported by transcriptomic analysis of differential gene expression during late-stage sporulation, in which a significant number of $B$. cereus-group specific genes of unknown function were severely down-regulated in the spoVT mutant strain. The potential role of one of these genes (BC1117) in sporulation was further investigated using a clean BC1117 deletion mutant strain. This strain sporulated normally, indicating that the sporulation phenotype observed in the $\Delta$ spoVT strain cannot be solely attributed to the extreme down-regulation of the $\mathrm{BC} 1117$ gene. The function of $\mathrm{BC} 1117$ and whether it plays a direct or indirect role in sporulation remains to be elucidated. Work is continuing to further characterize the BC1117 polypeptide in terms of its location and role in B. cereus spores.

Unfortunately, the synchronization of sporulation for RNA isolation proved challenging, limiting the usability and reliability of the obtained RNA sequencing data sets to time point T5 only. This could be the reason why only a limited number of known SpoVT regulon members were represented in our data set, which does not allow us to define the SpoVT regulon in $B$. cereus. Potentially, the optimization of sporulation protocols for $B$. cereus cells can help overcome this issue.

What can be derived from the transcriptomic data, however, is that the observed sporulation defect caused by lysis of formed prespores in $B$. cereus spoVT might be the result of premature germination of the prespores. Genes involved in both metabolism and protein synthesis were upregulated especially in the spoVT deletion strain, which is known to occur during the early stages of germination (Horsburgh et al., 2001). In addition, the spore cortex lytic enzyme (SCLE) CwlJ1 and the cortical fragment lytic enzyme (CFLE) SleL were upregulated over 5-fold and almost 4-fold, respectively, in a spoVT mutant of $B$. cereus. CwlJ is responsible for the degradation of the cortex layer upon germination and responds specifically to exogenous $\mathrm{Ca}^{2+}$ chelated dipicolinic acid (Ca-DPA) (Moir, 2006), whereas SleL cooperates with CwlJ by hydrolysing fragmented peptidoglycan substrates (Lambert et al., 2012), which supports the theory of premature germination in $B$. cereus $\Delta$ spoVT. Both genes encoding these proteins are known to be expressed in the mother cell under the control of $\sigma^{\mathrm{E}}$ and SpoIIID during early stages of sporulation. This suggests that the observed upregulation in a spoVT deletion mutant is more than likely an indirect effect. Such indirect effects may be more abundant, as several other regulatory proteins were also upregulated in the spoVT deletion strain (RsfA, SigK, GerE, and SigG). Together with the lack of a consensus SpoVT binding sequence, this complicates the determination of the SpoVT regulon in B. cereus. 
TABLE 3 | Significantly downregulated hypothetical genes in a B. cereus spoVT deletion mutant.

\begin{tabular}{lcl}
\hline Gene locus tag & Fold-change (down-regulated) & Protein homology \\
\hline BC1117 & 2984 & Hypothetical protein, homology to B. cereus group-specific hypothetical protein \\
BC0987 & 37 & Hypothetical protein, homology to hypothetical protein \\
BC1457 & 20 & Hypothetical protein, homology to hypothetical protein \\
BC2492 & 18 & Hypothetical protein, homology to B. cereus group-specific hypothetical protein \\
BC2270 & 15 & Hypothetical protein, homology to B. cereus group-specific hypothetical protein \\
BC3002 & 10 & Hypothetical protein, homology to B. cereus group-specific hypothetical protein \\
BC0973 & 9 & Hypothetical protein, B. cereus group membrane protein \\
BC2426 & 8 & Hypothetical protein, homology to B. cereus group-specific hypothetical protein \\
\hline
\end{tabular}

The heterogeneous character of SpoVT in sporulating bacteria is intriguing. Through this study we demonstrate an essential role of the SpoVT protein in sporulation of B. cereus, whereas in B. subtilis SpoVT is not essential, but nevertheless very important for proper formation and maturation of a spore. Further investigation on the essential and/or fine-tuning role of SpoVT in the regulation of sporulation-specific gene expression requires the quantification of active SpoVT levels, preferably in individual cells. This is currently complicated by the gaps in our knowledge on possible SpoVT activity requirements, e.g., the binding of a (yet unidentified) substrate. Obtaining such knowledge will also provide us with more insights on the role of SpoVT in sporulation and germination heterogeneity. Such phenotypic heterogeneity is an outcome of bifurcation in gene regulatory circuits, in which key regulatory proteins are often subjected to multimerization and require additional modification for activation before instigating feed forward loops in gene expression. In B. subtilis developmental processes, Spo0A and ComK are well-studied examples of such regulatory proteins (Grossman, 1995; Lopez et al., 2009). Given the requirement of tetramerization and possible substrate binding prior to activation, it is very plausible that SpoVT is equally involved in such complex regulatory circuits and is thus one of the major players responsible for germination heterogeneity. Levels of such a substrate may differ per individual cell, especially if the regulation of its production is part of a signal transduction system. Unfortunately, numerous attempts to identify such a substrate have thus far failed (Dong et al., 2004; Asen et al., 2009). New leads can be defined based on novel findings on the production of signaling molecules during sporulation, such as the recent demonstration of spore-specific production of cyclic di-AMP (c-di-AMP) via the CdaS cyclase during latestage sporulation (Mehne et al., 2013, 2014). Since the absence of CdaS in sporulating B. subtilis cells results in an affected germination profile of resulting spores (Mehne et al., 2014) and since cdaS deletion has a negative (direct or indirect) effect on sporulation gene expression levels (qPCR data not shown), it is tempting to assume that CdaS-derived c-di-AMP functions as a trigger molecule for SpoVT activity. Unfortunately, we have so far been unsuccessful in demonstrating an in vitro interaction between c-di-AMP and purified SpoVT $\mathrm{T}_{\mathrm{BSU}}$, whereas a clear interaction of c-di-AMP with the positive control DarA was observed (Jörg Stülke, personal communication, data not shown). Possibly, forespore-specific interactions between molecules are extra challenging to simulate in vitro (Asen et al., 2009), or c-di-AMP plays an important role during spore germination and/or outgrowth not related to SpoVT activity.

Differences in regulons of conserved regulatory proteins are not uncommon. In $B$. thuringiensis for instance, an organism closely related to $B$. cereus, it was found that the SinR regulatory protein was not involved in the regulation of an eps operon like in $B$. subtilis, but rather in the biosynthesis of the lipopeptide kurstakin and the $\mathrm{Hbl}$ enterotoxin gene that is not part of the B. subtilis genome (Fagerlund et al., 2014). In addition, for clostridial species it is well known that the regulatory programs orchestrating sporulation differ significantly from those in $B$. subtilis despite strong regulator protein conservation (Al-Hinai et al., 2015).

In summary, this report further demonstrates that despite conservation in sporulation regulatory proteins amongst Bacilli (de Hoon et al., 2010), future studies on their function and impact should take into account evolutionary differences in regulon members between species underlying important variances in downstream developmental processes.

\section{AUTHOR CONTRIBUTIONS}

RE designed the experimental plan, executed the majority of the experiments, analyzed the results, and wrote the manuscript. $\mathrm{SH}$ supported in the technical part and read the final manuscript. $\mathrm{AD}$ analyzed the transcriptomic results, contributed in writing, and read the final manuscript. AG executed the experiments with the BC1117 mutant, contributed in writing, and read the final manuscript. GC supported in the analysis of the BC1117 mutant results, contributed in writing, and read the final manuscript. OK participated in the experimental plan and read the final manuscript.

\section{FUNDING}

This research is funded by TI Food and Nutrition, a publicprivate partnership on pre-competitive research in food and nutrition. The funders had no role in study design, data 
collection and analysis, decision to publish, or preparation of the manuscript.

\section{ACKNOWLEDGMENTS}

We would like to thank Dr. Ruud Detert Oude Weme, Prof. Jörg Stülke and Christina Herzberg for technical assistance regarding $S_{\text {ppoVT }} B S U$ in vitro binding studies. We would also like to thank Prof. Simon Cutting for supplying us with the spoVT deletion strain of B. subtilis.

\section{SUPPLEMENTARY MATERIAL}

The Supplementary Material for this article can be found online at: http://journal.frontiersin.org/article/10.3389/fmicb. 2016.01607

\section{Figure S1 | Fluorescence microscopy analysis of cell sampling for} transcriptomic analysis. The sequential stages in sporulation were monitored in time using a fluorescing membrane dye. Time points (in hours) after initiation of sporulation are indicated.

Figure S2 | Light microscopy analysis of B. cereus ATCC 14579 cultures approximately $\mathbf{2 4} \mathrm{h}$ after entry to sporulation. Mature spores are evident in

\section{REFERENCES}

Abee, T., Groot, M. N., Tempelaars, M., Zwietering, M., Moezelaar, R., and van der Voort, M. (2011). Germination and outgrowth of spores of Bacillus cereus group members: diversity and role of germinant receptors. Food Microbiol. 28, 199-208. doi: 10.1016/j.fm.2010.03.015

Al-Hinai, M. A., Jones, S. W., and Papoutsakis, E. T. (2015). The Clostridium sporulation programs: diversity and preservation of endospore differentiation. Microbiol. Mol. Biol. Rev. 79, 19-37. doi: 10.1128/MMBR.00025-14

Arnaud, M., Chastanet, A., and Débarbouillé, M. (2004). New vector for efficient allelic replacement in naturally nontransformable, low-GC-content, gram-positive bacteria. Appl. Environ. Microbiol. 70, 6887-6891. doi: 10.1128/aem.70.11.6887-6891.2004

Asen, I., Djuranovic, S., Lupas, A. N., and Zeth, K. (2009). Crystal structure of SpoVT, the final modulator of gene expression during spore development in Bacillus subtilis. J. Mol. Biol. 386, 962-975. doi: 10.1016/j.jmb.2008.10.061

Augustin, J. C. (2011). Challenges in risk assessment and predictive microbiology of foodborne spore-forming bacteria. Food Microbiol. 28, 209-213. doi: 10.1016/j.fm.2010.05.003

Bagyan, I., Hobot, J., and Cutting, S. (1996). A compartmentalized regulator of developmental gene expression in Bacillus subtilis. J. Bacteriol. 178, 4500-4507.

Black, E. P., Linton, M., McCall, R. D., Curran, W., Fitzgerald, G. F., Kelly, A. L., et al. (2008). The combined effects of high pressure and nisin on germination and inactivation of Bacillus spores in milk. J. Appl. Microbiol. 105, 78-87. doi: 10.1111/j.1365-2672.2007.03722.x

Brown, K. L. (2000). Control of bacterial spores. Br. Med. Bull. 56, 158-171. doi: $10.1258 / 0007142001902860$

Carrera, M., Zandomeni, R. O., Fitzgibbon, J., and Sagripanti, J. L. (2007). Difference between the spore sizes of Bacillus anthracis and other Bacillus species. J. Appl. Microbiol. 102, 303-312. doi: 10.1111/j.1365-2672.2006.03111.x

Chary, V. K., Meloni, M., Hilbert, D. W., and Piggot, P. J. (2005). Control of the expression and compartmentalization of $\sigma^{\mathrm{G}}$ activity during sporulation of Bacillus subtilis by regulators of $\sigma^{\mathrm{F}}$ and $\sigma^{\mathrm{E}}$. J. Bacteriol. 187, 6832-6840. doi: 10.1128/jb.187.19.6832-6840.2005

Chen, F., Mackey, A. J., Stoeckert, C. J. Jr. and Roos, D. S. (2006). OrthoMCL-DB: querying a comprehensive multi-species collection of ortholog groups. Nucleic Acids Res. 34, D363-D368. doi: 10.1093/nar/gkj123 the (A) wild type and (C) $\triangle \mathrm{BC} 1117$ cultures, whereas the $\Delta$ SpoVT cells (B) have arrested during sporulation. Scale bar indicates $5 \mu \mathrm{M}$.

\section{Table S1 | Primers used in this study.}

Table S2 | Gene expression during sporulation in B. cereus and $B$ cereus $\Delta$ spoVT. Duplicate gene expression values during three time points in sporulation (T3, T4 and T5) for the wild type (WT) and the spoVT deletion strain (delta-VT) are indicated as reads per kilobase per million (rpkm).

Table S3 | Significant up-and downregulated genes in a spoVT deletion background of $\boldsymbol{B}$. cereus during T5. Down-regulation in gene expression in the spoVT-mutant strain is visualized in red, whereas up-regulation is visualized in green. The data for two biological replicates of the same strain are shown separately. The ratio in gene expression was calculated by taking the difference of the 2 log-transformed values at T5 between the wt and the spoVT mutant strain. The fold change in gene expression was calculated by raising the number 2 to the power of the absolute ratio value. Orthologs of known sporulation genes in B. subtilis are indicated in bold face. Regulation during sporulation in B. subtilis was derived from information on SporeWeb http://sporeweb.molgenrug.nl).

Movie S1 | Time-lapse microscopy movie for sporulating wild type $B$. cereus cells. The fluorescence shown in the right panel is derived from GFP expression driven by the $\mathrm{P}_{\text {spova }}$ promoter.

Movie S2 | Time-lapse microscopy movie for sporulating cells of $B$. cereus delta-spoVT. The fluorescence shown in the right panel is derived from GFP expression driven by the $\mathrm{P}_{\text {spova }}$ promoter.

Cronin, U. P., and Wilkinson, M. G. (2008). Bacillus cereus endospores exhibit a heterogeneous response to heat treatment and low-temperature storage. Food Microbiol. 25, 235-243. doi: 10.1016/j.fm.2007.11.004

de Hoon, M. J. L., Eichenberger, P., and Vitkup, D. (2010). Hierarchical evolution of the bacterial sporulation network. Curr. Biol. 20, R735-R745. doi: 10.1016/j.cub.2010.06.031

de Vries, Y. P., Hornstra, L. M., de Vos, W. M., and Abee, T. (2004). Growth and sporulation of Bacillus cereus ATCC 14579 under defined conditions: temporal expression of genes for key sigma factors. Appl. Environ. Microbiol. 70, 2514-2519. doi: 10.1128/AEM.70.4.2514-2519.2004

Dong, T. C., Cutting, S. M., and Lewis, R. J. (2004). DNA-binding studies on the Bacillus subtilis transcriptional regulator and AbrB homologue, SpoVT. FEMS Microbiol. Lett. 233, 247-256. doi: 10.1016/j.femsle.2004.02.013

Dworkin, J., and Shah, I. M. (2010). Exit from dormancy in microbial organisms. Nat. Rev.Microbiol. 8, 890-896. doi: 10.1038/nrmicro2453

Eijlander, R. T., Abee, T., and Kuipers, O. P. (2011). Bacterial spores in food: how phenotypic variability complicates prediction of spore properties and bacterial behavior. Curr. Opin. Biotechnol. 22, 180-186. 10.1016/j.copbio.2010.11.009

Eijlander, R. T., de Jong, A., Krawczyk, A. O., Holsappel, S., and Kuipers, O. P. (2014). SporeWeb: an interactive journey through the complete sporulation cycle of Bacillus subtilis. Nucleic Acids Res. 42, D685-D691. doi: 10.1093/nar/gkt1007

Eijlander, R. T., and Kuipers, O. P. (2013). Live-cell imaging tool optimization to study gene expression levels and dynamics in single cells of Bacillus cereus. Appl. Environ. Microbiol. 79, 5643-5651. doi: 10.1128/aem.01347-13

Fagerlund, A., Dubois, T., Økstad, O. A., Verplaetse, E., Gilois, N., Bennaceur, I., et al. (2014). SinR controls enterotoxin expression in Bacillus thuringiensis biofilms. PLoS ONE 9:e87532. doi: 10.1371/journal.pone.0087532

Galperin, M. Y., Mekhedov, S. L., Puigbo, P., Smirnov, S., Wolf, Y. I., and Rigden, D. J. (2012). Genomic determinants of sporulation in Bacilli and Clostridia: towards the minimal set of sporulation-specific genes. Environ. Microbiol. 14, 2870-2890. doi: 10.1111/j.1462-2920.2012.02841.x

Grossman, A. D. (1995). Genetic networks controlling the initiation of sporulation and the development of genetic competence in Bacillus subtilis. Annu. Rev. Genet. 29, 477-508. doi: 10.1146/annurev.ge.29.120195.002401

Guérout-Fleury, A. M., Shazand, K., Frandsen, N., and Stragier, P. (1995). Antibiotic-resistance cassettes for Bacillus subtilis. Gene 167, 335-336. 
Hilbert, D. W., and Piggot, P. J. (2004). Compartmentalization of gene expression during Bacillus subtilis spore formation. Microbiol. Mol. Biol. Rev. 68, 234-262. doi: 10.1128/MMBR.68.2.234-262.2004

Hornstra, L. M., de Vries, Y. P., de Vos, W. M., Abee, T., and Wells-Bennik, M. H. J. (2005). gerR, a novel ger operon involved in l-alanine- and inosineinitiated germination of Bacillus cereus ATCC 14579. Appl. Environ. Microbiol. 71, 774-781. doi: 10.1128/AEM.71.2.774-781.2005

Horsburgh, M. J., Thackray, P. D., and Moir, A. (2001). Transcriptional responses during outgrowth of Bacillus subtilis endospores. Microbiology 147, 2933-2941. doi: 10.1099/00221287-147-11-2933

Janes, B. K., and Stibitz, S. (2006). Routine markerless gene replacement in Bacillus anthracis. Infect. Immun. 74, 1949-1953. doi: 10.1128/IAI.74.3.1949-1953.2006

Kunst, F., Ogasawara, N., Moszer, I., Albertini, A. M., Alloni, G., Azevedo, V., et al. (1997). The complete genome sequence of the Gram-positive bacterium Bacillus subtilis. Nature 390, 249-256.

Lambert, E. A., Sherry, N., and Popham, D. L. (2012). In vitro and In vivo analyses of the Bacillus anthracis spore cortex lytic protein SleL. Microbiology 158, 1359-1368. doi: 10.1099/mic.0.056630-0

Langmead, B., and Salzberg, S. L. (2012). Fast gapped-read alignment with Bowtie 2. Nat. Methods 9, 357-359. doi: 10.1038/nmeth.1923

Lewis, P. J., and Marston, A. L. (1999). GFP vectors for controlled expression and dual labelling of protein fusions in Bacillus subtilis. Gene 227, 101-110.

Li, H., Handsaker, B., Wysoker, A., Fennell, T., Ruan, J., Homer, N., et al. (2009). The sequence alignment/map format and samtools. Bioinformatics 25, 2078-2079. doi: 10.1093/bioinformatics/btp352

Li, Z., and Piggot, P. J. (2001). Development of a two-part transcription probe to determine the completeness of temporal and spatial compartmentalization of gene expression during bacterial development. Proc. Natl Acad. Sci. U.S.A. 98, 12538-12543. doi: 10.1073/pnas.221454798

Lindbäck, T., Mols, M., Basset, C., Granum, P. E., Kuipers, O. P., and Kovács, A. T. (2012). CodY, a pleiotropic regulator, influences multicellular behaviour and efficient production of virulence factors in Bacillus cereus. Environ. Microbiol. 14, 2233-2246. doi: 10.1111/j.1462-2920.2012.02766.x

Logan, N. A. (2012). Bacillus and relatives in foodborne illness. J. Appl. Microbiol. 112, 417-429. doi: 10.1111/j.1365-2672.2011.05204.x

Lopez, D., Vlamakis, H., and Kolter, R. (2009). Generation of multiple cell types in Bacillus subtilis. FEMS Microbiol. Rev. 33, 152-163. doi: 10.1111/j.15746976.2008.00148.x

Lücking, G., Stoeckel, M., Atamer, Z., Hinrichs, J., and Ehling-Schulz, M. (2013). Characterization of aerobic spore-forming bacteria associated with industrial dairy processing environments and product spoilage. Int. J. Food Microbiol. 166, 270-279. doi: 10.1016/j.ijfoodmicro.2013.07.004

Masson, L., Préfontaine, G., and Brousseau, R. (1989). Transformation of Bacillus thuringiensis vegetative cells by electroporation. FEMS Microbiol. Lett. 51, 273-277.

Mehne, F. M., Gunka, K., Eilers, H., Herzberg, C., Kaever, V., and Stülke, J. (2013). Cyclic di-AMP homeostasis in Bacillus subtilis: both lack and high level accumulation of the nucleotide are detrimental for cell growth. J. Biol. Chem. 288, 2004-2017. doi: 10.1074/jbc.M112.395491

Mehne, F. M., Schröder-Tittmann, K., Eijlander, R. T., Herzberg, C., Hewitt, L., Kaever, V., et al. (2014). Control of the diadenylate cyclase CdaS in Bacillus subtilis: an autoinhibitory domain limits cyclic di-AMP production. J. Biol. Chem. 289, 21098-21107. doi: 10.1074/jbc.M114.562066

Moir, A. (2006). How do spores germinate? J. Appl. Microbiol. 101, 526-530. doi: $10.1111 / \mathrm{j} .1365-2672.2006 .02885 . \mathrm{x}$

Økstad, O. A., and Kolstø, A. B. (2011). "Food microbiology and food safety," in Genomics of Foodborne Bacterial Pathogens, Vol. 29, eds M. Wiedmann and W. Zhang (Oslo: Springer Science + Business Media, LLC), 29-53. doi: 10.1007/978-1-4419-7686-4_2

Pflughoeft, K. J., Sumby, P., and Koehler, T. M. (2011). Bacillus anthracis sin locus and regulation of secreted proteases. J. Bacteriol. 193, 631-639. doi: 10.1128/JB.01083-10
Piggot, P. J., and Hilbert, D. W. (2004). Sporulation of Bacillus subtilis. Curr. Opin. Microbiol. 7, 579-586. doi: 10.1016/j.mib.2004.10.001

Qin, H., and Driks, A. (2013). Contrasting evolutionary patterns of spore coat proteins in two Bacillus species groups are linked to a difference in cellular structure. BMC Evol Biol. 13:261. doi: 10.1186/1471-2148-13-261

Ramirez-Peralta, A., Stewart, K. A., Thomas, S. K., Setlow, B., Chen, Z., Li, Y. Q., et al. (2012). Effects of the SpoVT regulatory protein on the germination and germination protein levels of spores of Bacillus subtilis. J. Bacteriol. 194, 3417-3425. doi: 10.1128/jb.00504-12

Rudner, D. Z., and Losick, R. (2001). Morphological coupling in development: lessons from prokaryotes. Dev. Cell 1, 733-742. doi: 10.1016/S15345807(01)00094-6

Sella, S. R., Vandenberghe, L. P., and Soccol, C. R. (2014). Life cycle and spore resistance of spore-forming Bacillus atrophaeus. Microbiol. Res. 169, 931-939. doi: 10.1016/j.micres.2014.05.001

Setlow, P. (2003). Spore germination. Curr. Opin. Microbiol. 6, 550-556.

Setlow, P. (2006). Spores of Bacillus subtilis: their resistance to and killing by radiation, heat and chemicals. J. Appl. Microbiol. 101, 514-525. doi: 10.1111/j.1365-2672.2005.02736.x

Setlow, P. (2007). I will survive: DNA protection in bacterial spores. Trends Microbiol. 15, 172-180. doi: 10.1016/j.tim.2007.02.004

Setlow, P. (2013). Summer meeting 2013 - when the sleepers wake: the germination of spores of Bacillus species. J. Appl. Microbiol. 115, 1251-1268. doi: 10.1111/jam.12343

Stenfors Arnesen, L. P., Fagerlund, A., and Granum, P. E. (2008). From soil to gut: Bacillus cereus and its food poisoning toxins. FEMS Microbiol. Rev. 32, 579-606. doi: 10.1111/j.1574-6976.2008.00112.x

Trapnell, C., Roberts, A., Goff, L., Pertea, G., Kim, D., Kelley, D. R., et al. (2012). Differential gene and transcript expression analysis of RNA-seq experiments with TopHat and Cufflinks. Nat. Protoc. 7, 562-578. doi: 10.1038/nprot.2012.016

Turgeon, N., Laflamme, C., Ho, J., and Duchaine, C. (2008). Evaluation of the plasmid copy number in $B$. cereus spores, during germination, bacterial growth and sporulation using real-time PCR. Plasmid 60, 118-124. doi: 10.1016/j.plasmid.2008.05.001

van der Voort, M., García, D., Moezelaar, R., and Abee, T. (2010). Germinant receptor diversity and germination responses of four strains of the Bacillus cereus group. Int. J. Food Microbiol. 139, 108-115. doi: 10.1016/j.ijfoodmicro.2010.01.028

Wang, J. C., Hu, S. H., and Lin, C. Y. (2003). Lethal effect of microwaves on spores of Bacillus spp. J. Food Prot. 66, 604-609.

Wang, R., Krishnamurthy, S. N., Jeong, J. S., Driks, A., Mehta, M., and Gingras, B. A. (2007). Fingerprinting species and strains of Bacilli spores by distinctive coat surface morphology. Langmuir 23, 10230-10234. doi: 10.1021/la701788d

Wang, S. T., Setlow, B., Conlon, E. M., Lyon, J. L., Imamura, D., Sato, T., et al. (2006). The forespore line of gene expression in Bacillus subtilis. J. Mol. Biol. 358, 16-37. doi: 10.1016/j.jmb.2006.01.059

Yudkin, M. D., and Clarkson, J. (2005). Differential gene expression in genetically identical sister cells: the initiation of sporulation in Bacillus subtilis. Mol. Microbiol. 56, 578-589. doi: 10.1111/j.1365-2958.2005.04594.x

Conflict of Interest Statement: The authors declare that the research was conducted in the absence of any commercial or financial relationships that could be construed as a potential conflict of interest.

Copyright (c) 2016 Eijlander, Holsappel, de Jong, Ghosh, Christie and Kuipers. This is an open-access article distributed under the terms of the Creative Commons Attribution License (CC BY). The use, distribution or reproduction in other forums is permitted, provided the original author(s) or licensor are credited and that the original publication in this journal is cited, in accordance with accepted academic practice. No use, distribution or reproduction is permitted which does not comply with these terms. 